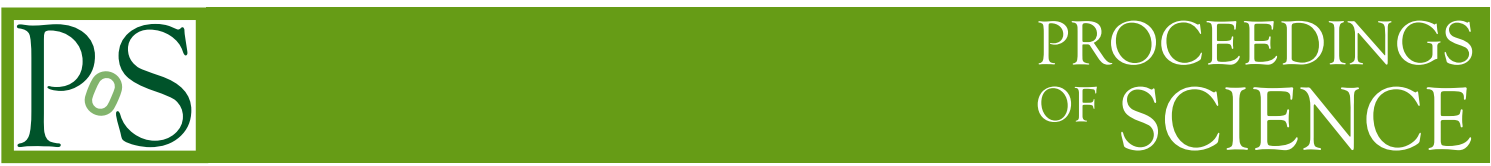

\title{
Gamma rays from $\eta$ Carinae
}

\section{Matteo Balbo*}

Department of Astronomy, University of Geneva, Chemin d'Ecogia 16, 1290 Versoix, Switzerland

E-mail: Matteo.Balbo@unige.ch

\section{Roland Walter}

Department of Astronomy, University of Geneva, Chemin d'Ecogia 16, 1290 Versoix, Switzerland

E-mail: Roland.Walter@unige.ch

The $\eta$ Carinae binary system is the first $\gamma$-ray binary ever observed which does not contain a compact object. It can be considered as a natural laboratory to study particle acceleration and $\gamma$-ray emission. Indeed the dense wind of the primary star shocks against the fast light wind coming from the companion star, creating the conditions to accelerate particles up to relativistic energies via Fermi mechanism. These relativistic particles subsequently dissipate energy as nonthermal radiation. Fermi-LAT and H.E.S.S. detections of $\eta$ Carinae confirm such hypotheses.

Hydrodynamic simulations provide a convincing match to the observations if a few percent of the wind mechanical energy dissipated in the shock goes into particle acceleration. The intrinsic $\pi^{0}$ decay spectrum is a complex convolution of the maximum energy, luminosity, particle drift and obscuration. Accelerated particles cool down mainly via inverse-Compton, synchrotron radiation, and proton-proton collisions. High-energy $\gamma$ rays interact also with the field of anisotropic UV photons emitted by both luminous stars, creating $e^{ \pm}$pairs and strongly modifying the observed spectrum. Quick variations of the optical depth are expected along the orbit, due to changes in shape, position, and gas density of the shocked region. Various CTA simulations confirm that flux variability down to few days timescale could be detected above $30 \mathrm{GeV}$. These variations could disentangle the intrinsic particle spectral cut off from that related to $\gamma$ - $\gamma$ opacity and determine the flux of relativistic protons and positrons injected in the interstellar medium, the geometry of the colliding wind region and the magnetic field configuration, as well as the geometrical orientation of the binary system (although not without some assumptions). CTA will also enlighten the nature of the high-energy component, the mechanisms and the percentage of kinetic energy channelled into particle acceleration.

High Energy Phenomena in Relativistic Outflows VII - HEPRO VII

9-12 July 2019

Facultat de Física, Universitat de Barcelona, Spain

${ }^{*}$ Speaker. 


\section{Colliding wind binaries}

A colliding wind binary (CWB) is a binary star system consisting of two non-compact objects, likely OB-type or Wolf-Rayet (WR) stars. WRs represent the final stage of the evolution of the most massive stars, before ending their lives as supernovae. Such stars possess the highest known mass loss rates $\left(10^{-8} \sim 10^{-3} \mathrm{M}_{\odot} \mathrm{yr}^{-1}\right)$, and their winds can reach terminal velocities $\left(v_{\infty}\right)$ up to few thousands $\mathrm{km} \mathrm{s}^{-1}[1,2]$. The kinetic energy of the supersonic wind $L_{w}=\dot{M} v_{\infty}^{2} / 2$ rarely exceeds $1 \%$ of the bolometric radiative energy output, which typically is of the order of $\sim 10^{38} \mathrm{erg} \mathrm{s}^{-1}$. The radiation pressure drives those powerful winds and creates two strong shock fronts, where the compressed plasma heats up to $\sim 10^{7} \mathrm{~K}$ and emits mainly in X-rays [3]. In addition to the thermal $\mathrm{X}$-rays emitted in the strong shocks where winds collide, in these regions particles can potentially reach high energies (HE) via diffusive shock acceleration (DSA) [4, 5], and they can be exposed to high magnetic field from the hot stars. The existence of the star magnetic field is confirmed by the detected radio synchrotron emission [6]. The relativistic particles can also interact with the large pool of soft photons (from IR to UV), which are up-scattered via inverse-Compton (IC) to the $\mathrm{GeV}$ energy band, cooling the energetic particles. Other possible cooling mechanisms are Bremsstrahlung, pp collisions or synchrotron radiation [7].

\section{2. $\eta$ Carinae: a PeVatron laboratory}

The $\eta$ Carinae $^{1}$ binary system raised quite some interest in the recent years, after becoming the first binary system ever detected emitting HE $\gamma$ rays, without hosting a compact object. Its main star is supposed to be one of the most massive and luminous star of our Galaxy, and is thought to be a Luminous Blue Variable (LBV). Its initial mass is expected to be $\gtrsim 90 M_{\odot}$ [8], possibly even up to $\sim 200 \mathrm{M}_{\odot}$. For many centuries it was thought to be a single star, and its optical brightness was showing several fluctuations, with a gradually increase up to $18^{\text {th }}$ century. Starting from this century, some recorded data are available to trace a light curve (LC) [9]. Nowadays such fluctuations are generally associated with pre-eruption shifts in apparent temperature of the star's wind, rather than an intrinsic change in luminosity [10]. Observations by Sir John Herschel [11], confirmed that $\eta$ Carinae underwent the so-called Great Eruption between 1837 and 1858, increasing its luminosity by more than a factor ten ${ }^{2}$, reaching a total luminosity of the order of $10^{7.3} \mathrm{~L}_{\odot}$. During this $\sim 20$ year eruption $\eta$ Carinae ejected an amount of mass of about 10 to $40 M_{\odot}[12]$ at an average speed of $\sim 650 \mathrm{~km} \mathrm{~s}^{-1}$ [13]. The released kinetic energy of the ejected material was $\sim 10^{49.7} \mathrm{ergs}$, similar to its bolometric luminosity. Such great eruption is at the origin of the Homunculus Nebula, consisting of two pronounced lobes and a large thin equatorial disk.

A second eruption occurred also between 1887 and 1895, but the released amount of material $\left(\sim 0.2 \mathrm{M}_{\odot}\right)$ and its kinetic energy this time was smaller $\left(10^{46.9} \mathrm{erg}\right)$, while its bolometric luminosity was $10^{48.6} \mathrm{erg}$. Thus $\eta$ Carinae ejected material in the polar and equatorial directions during both eruptions, but with different energies and very likely different physical causes.

\footnotetext{
${ }^{1}$ The first (optical) observation of the system dates back to 1603, and its original name was $\eta$ Argus.

${ }^{2}$ The Great Eruption was almost as bright as a supernova explosion, but contrarily to such type of event, the binary survived. This is why sometimes $\eta$ Carinae is also nicknamed as "supernova impostor".
} 
It was not until the last decades of the century, that it was possible to discover the existence of the companion star, and consequently the binarity of the system and its periodicity of 5.52 year [14]. The companion star remains unobserved up to now at any wavelength, but it is supposed to be of O-type or may be a WR.

The LBV is accelerating a very dense wind with a mass loss rate of $\sim 8.5 \times 10^{-4} \mathrm{M}_{\odot} \mathrm{yr}^{-1}$ and a slow terminal velocity of $\sim 420 \mathrm{~km} \mathrm{~s}^{-1}$ [15]. The WR also emits a powerful wind, less dense but faster, with mass loss rates of $\simeq 10^{-5} \mathrm{M}_{\odot} \mathrm{yr}^{-1}$ and velocities up to $\sim 3000 \mathrm{~km} \mathrm{~s}^{-1}$ [16]. X-ray observations of the system have been regularly performed since 1997, with the Rossi X-ray Timing Explorer (RXTE) [17] with nearly weekly intervals (up to daily frequencies during important events), which have refined the estimated ${ }^{3}$ periodicity to $2024 \pm 2$ days [18]. The modulation detected in the X-ray band LC indicates that the two stars are on a very eccentric orbit with $e \simeq 0.9 \sim 0.95[19]$.

\section{Orbital variability: from $\mathrm{X}$-ray band to $\mathrm{GeV}$}

The high estimated eccentricity of the $\eta$ Carinae orbit implies that the relative separation of the two stars varies by a factor $\sim 10-20$ during a full period. At periastron, the two objects pass within a few $\mathrm{AU}$ of each other, a distance just a few times larger than the size of the primary star. In the colliding wind region (CWR) the accelerated particles encounter conditions that vary along the orbit: particle density, pressure, gas temperature, local magnetic field, separation between the two stars, etc. (see Fig. 1), so an orbital dependency of the $\gamma$-ray emission is expected.

The collision of the two stellar winds explains well the X-ray emission modulated by the orbit. The shocked gas can cool by expansion flowing away from the stagnation point along the contact discontinuity, or by radiation. During different orbital phases, the cooling can be dominated by expansion or by radiation. In the latter case, the shock structure becomes unstable [21], something expected to happen when $\eta$ Carinae is close to periastron [20]. Another aspect in favour of the CWR disruption scenario is the high orbital velocity with which the companion approaches the main star during periastron $\left(300 \sim 400 \mathrm{~km} \mathrm{~s}^{-1}\right.$ ) combined with the small separation between the two stars.

Some cycle-to-cycle variations are present around different periastron passages [22]. This is particularly evident in the 2009 periastron event, when the recovery from the X-ray minimum lasted only 1.5 months (nearly half of the usual duration), and in the 2014.5 periastron passage, when the $\mathrm{X}$-ray maximum reached a significantly higher flux. Such variation could be interpreted as small scale variation of the geometry of the shock winds, or with a lower/higher clumpiness degree of the two interacting winds. Cooling processes reduce the downwind gas pressure, shrinking the shocked gas into a thinner layer. The plasma flowing over this thin layer can encounter transverse acceleration instabilities [23] and/or thin-layer instabilities [24]. The observed long-term X-ray modulation, instead, is eventually associated with the time evolution of the ejecta.

The hard X-ray emission detected by INTEGRAL [25] and Suzaku [19], with an average luminosity $(\sim 4-7) \times 10^{33} \mathrm{erg} \mathrm{s}^{-1}$, suggested the presence of relativistic particles in the system. With Fermi-LAT we detected two components in the spectrum around periastron passage of 2009

\footnotetext{
${ }^{3}$ The orbital period at the epoch of the Great Eruption was estimated to be around $\sim 5.1 \mathrm{yr}$.
} 


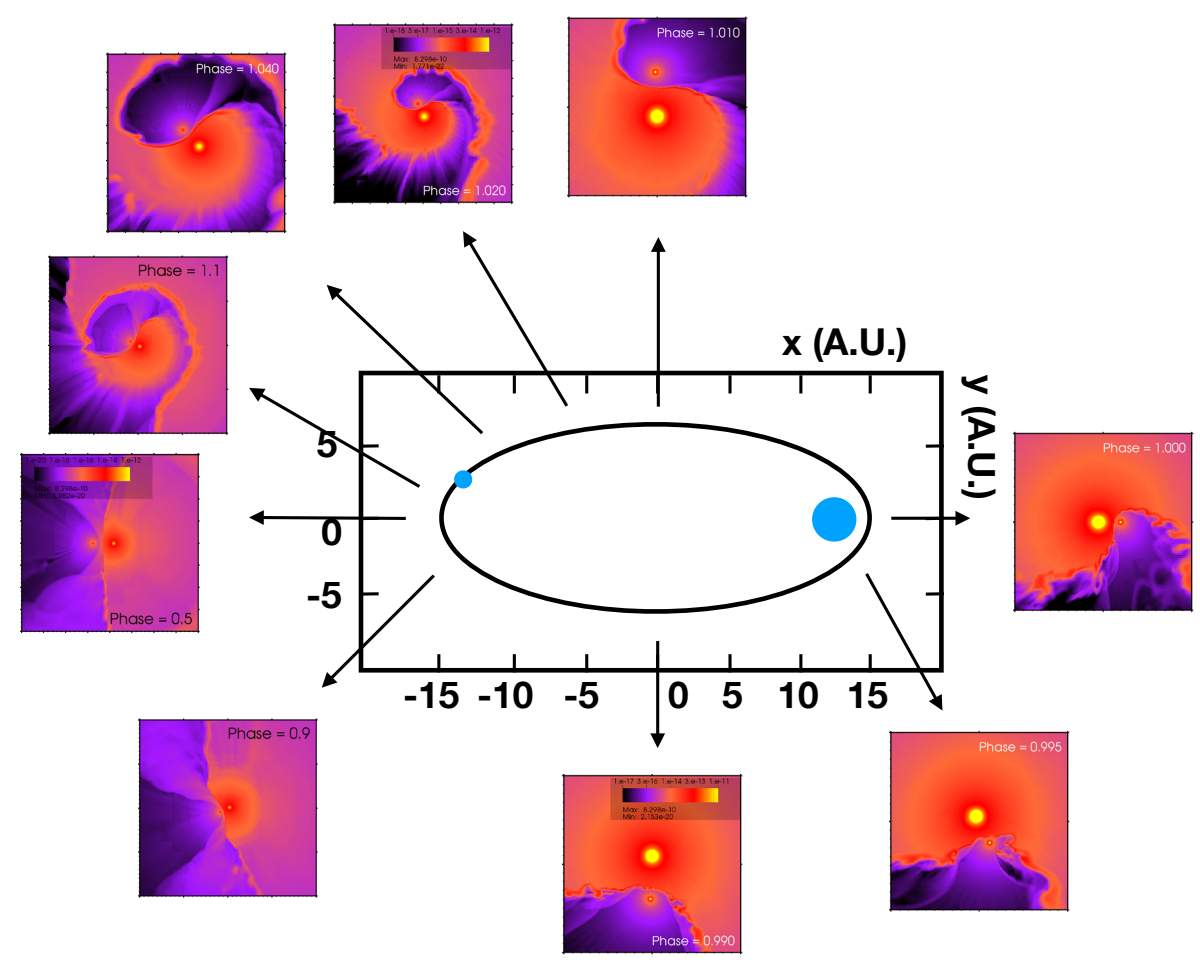

Figure 1: Different hydro-dynamical simulations of [20] for different orbital phases of $\eta$ Carinae.

[26], associating the low energy (LE) component with the IC emission, which cuts off at around few $\mathrm{GeV}$, and well correlates with the orbital motion. Instead, the most energetic component is likely attributed to $\pi^{0}$ decay (resulting from $p p$ collisions) and can be strongly modulated by $\gamma-\gamma$ absorption. Its emission varies by a factor $\sim 3-4$ from periastron to apastron, but the limited statistics of the data does not show any evident correlation with the orbital phase. Cherenkov observations also confirmed the detection of $\eta$ Carinae in the highest energy part of the spectrum [27], and implies a sudden drop around $\gtrsim 1 \mathrm{TeV}$. This could be interpreted either as a cut off in the intrinsic accelerated particle distribution or due to severe $\gamma-\gamma$ absorption.

Using the hydro-dynamical simulation results which model the interacting stellar winds at each orbital phase [20], we have calculated the shock velocities, the mechanical power, and the maximum energy that particles could reach in each adaptive cell of the simulation, balancing the different radiative cooling times (synchrotron, IC, Bremsstrahlung, etc.) with the characteristic acceleration time of the DSA.

Most of the shock power is released on both sides of the wind collision zone and in the cells downstream the CWR [28]. The increasing shock area compensates for the loss of the released energy density up to a relatively large distance from the centre of mass, explaining why the X-ray luminosity at apastron is still about a third of the peak emission at periastron. Computing the emissivity of the resulting electron spectra, we could reproduce the LE (0.3-10 GeV) $\gamma$-ray LC of $\eta$ Carinae very well (see Fig. 2a), with the only overestimation of a time interval after the periastron, which suggests that the shock front can be more unstable. In particular, the "reverse 


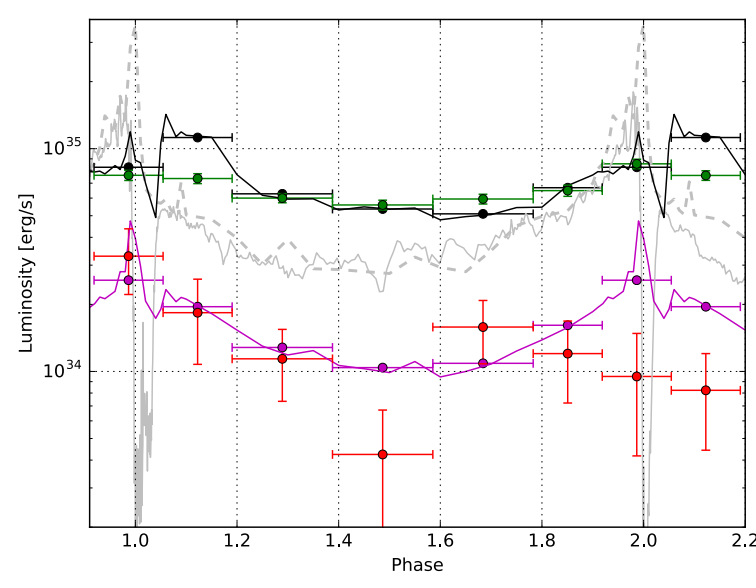

(a)

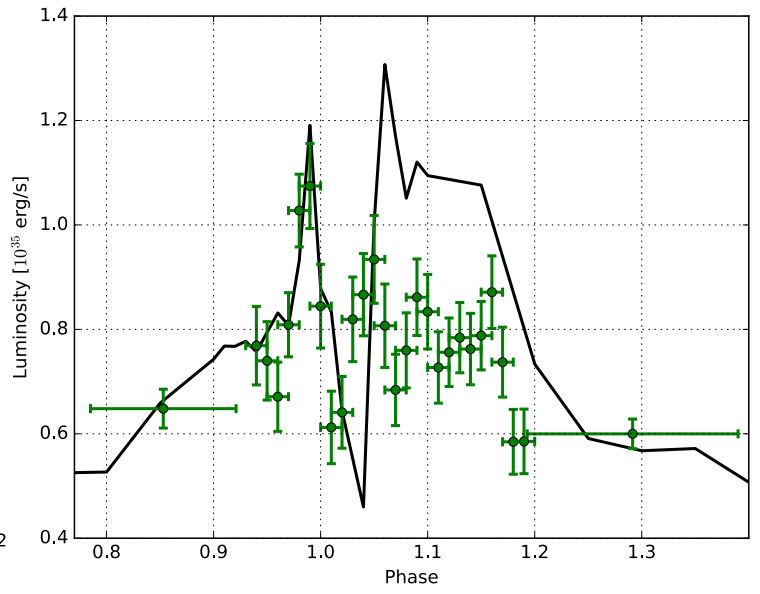

(b)

Figure 2: (a) Simulated and observed X-ray and $\gamma$-ray LCs of $\eta$ Carinae. The black and purple lines and bins show the predicted IC and $\pi^{0}$ decay LCs. The green and red points show the observed Fermi-LAT LC at low (0.3-10 GeV) and high (10-300 GeV) energies. The dim gray lines show the observed (continuous) and predicted (dash, without obscuration) thermal X-ray LCs. Errorbars are $1 \sigma$. (b) A merged Fermi-LAT analysis $(0.3-10 \mathrm{GeV})$ of the two periastrons for narrow time bins. The two broad bins and the black curve are the same as in Fig. 2a.

bubble cavity" (see Fig. 1) that is created by the close passage of the companion with its very fast wind could collapse. The relative importance of this secondary peak depends on the magnetic field geometry, radiation transfer, obscuration and other details of the hydro-dynamics, and its spectral shape could be different. The fact that the LE $\gamma$-ray LC showed a similar behaviour during both periastrons, allowed us to perform a stacked analysis below $\lesssim 10 \mathrm{GeV}$, decreasing the temporal resolution down to $\sim 40$ days (see Fig. $2 b$ ).

The mechanical luminosity available in the shock increases towards periastron. The same trend is followed by the thermal emission and by the LE $\gamma$ rays, almost doubling in the phase range $\approx 1.05-1.15$. The latter peak corresponds to the "reverse bubble", which effectively doubled the shock front area during about a tenth of the orbit [20]. The mechanical luminosity shows also a local minimum between phases 1.0 and 1.05, when the central part of the CWR is disrupted, similarly to the X-ray emission. IC cooling can not explain any prominent feature in the SED, except if the seed electrons from the two winds present similar luminosities, but are accelerated to significantly different maximum energies [26]. So leptons can hardly explain the VHE $\gamma$-ray emission observed during 2009 periastron passage. Furthermore, their contribution seems to cut off at around few $\mathrm{GeV}$ (see Fig. 3).

The hard component above $\gtrsim 10 \mathrm{GeV}$ could be explained uniquely with a hadronic contribution, and its emission takes place close to the center. Results of simulations suggest a surface magnetic field in the range $\sim 0.4-1 \mathrm{kG}$, which in turn can be further reduced depending on the magnetic field amplification factor assumed in the strong shocks [30]. Particle acceleration in turbulent reconnection at the current sheets is not excluded [31] and is favoured by small-scale turbulent motions [32]. Assuming that $\sim 2.4 \%$ of the mechanical energy goes into proton acceler- 


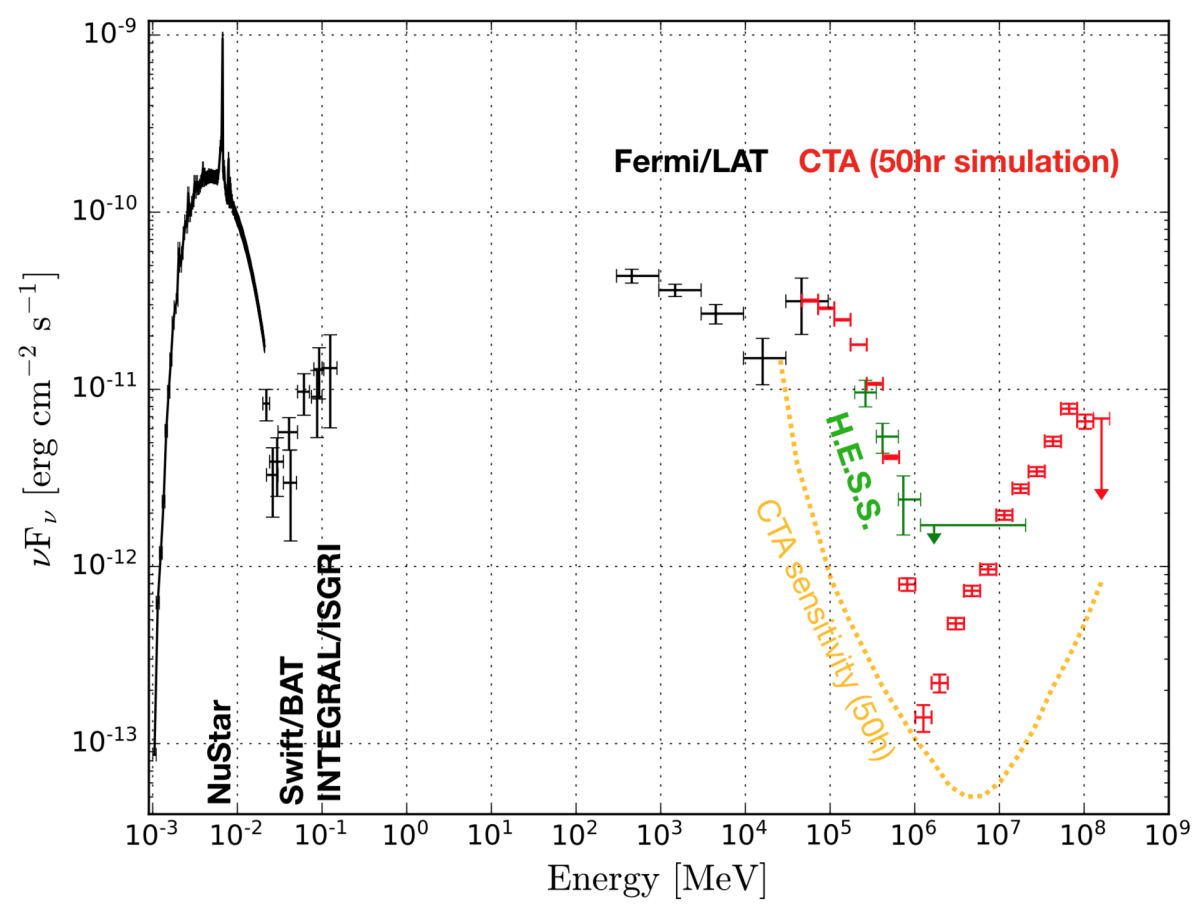

Figure 3: Spectral energy distribution of $\eta$ Carinae from $1 \mathrm{keV}$ to $100 \mathrm{TeV}$. The data are from NuStar [29], Swift, INTEGRAL, Fermi-LAT and H.E.S.S. and obtained close to periastron. The red points show the results of a simulation of what could be detected by CTA (at periastron) assuming that the emission is dominated by $\pi^{0}$ decay modified by $\gamma-\gamma$ absorption in the strong ultraviolet photon field.

ation, $\gamma$ rays emitted by $\pi^{0}$ decay could well reproduce the variability pattern from 2009 periastron toward 2012 apastron. At periastron, protons could reach energy close to $10^{15} \mathrm{eV}$ (i.e. close to the knee of the cosmic-ray spectrum). Differently from SNRs that can efficiently accelerate particles only for a limited period of their evolution (few $\sim 10^{3}$ years), CWBs can accelerate particles for a much longer period of their life, providing even up to $10^{48 \sim 49} \mathrm{erg}$ of cosmic-ray energy.

Despite the variability from 2009 periastron to 2012 apastron was well predicted by the simulation, in the following 2014.5 periastron passage the hard component did not show up again, remaining at a flux level compatible with the apastron. The intrinsic $\pi^{0}$ decay spectrum is a complex convolution of the maximum energy, luminosity, particle drift and obscuration expected in every simulation cell. The scarce statistics of events at HE does not allow to reduce the temporal binning of the LC much below several months.

The fraction of the shock mechanical luminosity accelerating electrons appears to be slightly smaller than the one that accelerates protons. These results contrast with the efficiencies derived from the latest PIC simulations [33], involving low magnetic fields, radiation and particle densities and favouring hadronic acceleration in the context of SNR. Purely hadronic acceleration has been proposed by [34] to explain the GeV spectrum of $\eta$ Carinae. In that case the two spectral components are related to the different hadron interaction times observed on the two sides of the wind 
separation surface, largely because of the contrast in density and magnetic field. Similarly to what happens to electrons, in our simulations this effect is smoothed by the many zones of the model, each characterized by different conditions. Even if the shock on the companion side does contribute more at high energies, the resulting $\pi^{0}$ decay spectrum does not feature two distinct components. An instrument sensitive in the LE $\gamma$-ray band (1-100 MeV) would easily discriminate between the lepto-hadronic and purely hadronic models. Indeed, the IC $\gamma$-ray emission of the primary electron component would be much stronger than the one predicted by secondary leptons produced in the purely hadronic acceleration scenario.

\section{Opacity study for high-energy $\gamma$ rays and prospects for CTA}

The multiwavelength spectrum of $\eta$ Carinae is quite well understood, from the radio to the $\mathrm{X}$-ray band. Instead, for energies above the $\mathrm{MeV}$ domain the correct interpretation of the spectrum is still debated, mostly due to the very low statistics of the available data. The clear presence of a hard component around 2009 periastron above $\gtrsim 10 \mathrm{GeV}$ can hardly be explained invoking a leptonic scenario [26]. The observed feature around $\sim 10 \mathrm{GeV}$ would require an ad hoc distribution of X-ray photons to play a significant role with the keV-GeV photon absorption [35]. In fact, the opacities measured for X-ray and $\gamma$-ray photons in our simulation [26], along different possible lines of sight, yield always a negligible value of $\tau<10^{-2}$ around periastron, and many orders of magnitude smaller during all other phases.

The production of $\gamma$ rays could potentially derive from $p \gamma$ or $p p$ interactions. The former presents a cross section two orders of magnitude smaller than the latter one, and its peak occurs when $E_{\gamma} \cdot E_{p} \simeq 3.5 \times 10^{17} \mathrm{eV}^{2}$. As the maximum of the thermal soft photons is emitted in the UV energy band, the required proton energy would result to be extremely high $E_{p} \simeq 10 \sim 100 \mathrm{PeV}$, thus making such process very unlikely. Less energetic protons instead would be required if the interactions happen with more energetic $\gamma$ rays (e.g. those UV that are up-scattered via IC scattering off relativistic electrons), but the $p p$ channel remains the most likely one.

Above several hundreds of $\mathrm{GeV}, \gamma-\gamma$ absorption and subsequent $e^{ \pm}$pairs production could arise from $\mathrm{eV}-\mathrm{TeV}$ photon interactions, making the photon field opaque to $\sim \mathrm{TeV}$ photons. The region is indeed constantly replenished by thermal UV photons from the massive star, whose density reduces for higher distances $\propto 1 / r^{2}$. The obscuration is likely maximized at periastron, when the ultraviolet photon field is particularly dense and the intrinsic cut off energy of the particle spectra is the highest. The probability of interaction for the two photons depends on the density of the UV and $\gamma$ rays, on the scattering angle between the two interacting photons, and on the orientation of the system with respect to the observer. The location where relativistic $\gamma$ rays are produced with respect to the main star and the observer changes as a direct consequence of the orbital motion. So HE photons travelling toward us can interact along their path with UV photons at angles which vary according to the different orbital phases. For a given UV photon of energy $\sim k_{B} T$ and a $\gamma$ ray of energy $E_{\gamma}$, the $\gamma-\gamma$ absorption can be expressed as a function of $\xi=E_{\gamma} \cdot k_{B} T /\left(m_{e} c^{2}\right)^{2}$. If collisions occur mostly head-on, the peak of the photon-photon absorption is located around $\xi \simeq 1.4$, whereas in case of tail-in collisions it moves towards $\xi \simeq 30$ [36].

Using the most likely orientation of the binary system [37], the strongest absorption variability is supposed to occur around periastron. Approaching periastron, indeed, the companion star 


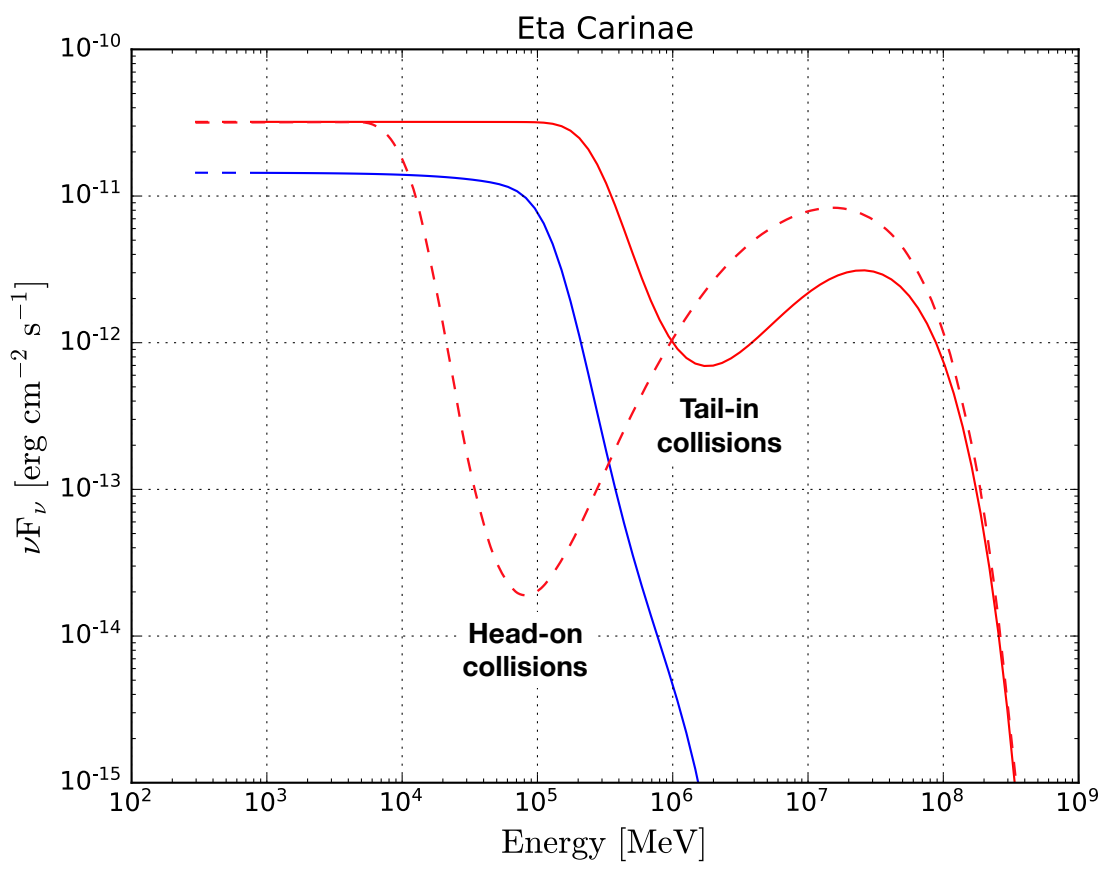

Figure 4: Possible hadronic $\gamma$-ray emission from $\eta$ Carinae, convoluted with the expected $\gamma-\gamma$ absorption and the different particle energy cut off, at around periastron (red) and apastron (blue). The dashed line shows the theoretical variability expected at around periastron [38].

lies between the main star and the observer. Once periastron is passed, the companion star moves further away from the observer and the main star. The two stars are roughly aligned at phases 0.9 and 1.1. The HE photons produced by the colliding winds will propagate towards the observer initially interacting with the strong ultraviolet field, mostly with "tail-in" collisions. Once periastron is passed, the collisions will mainly occur "head-on" from the shocked region up to the main star, and "tail-in" when the photons will propagate further. The final $\gamma-\gamma$ absorption varies by nearly a factor 40 in intensity when collisions pass from "head-on" to "tail-in" [34]. Furthermore, the energy of the maximum absorption varies by a factor $>20$. Assuming a black-body emission for the main star with a temperature of $37000 \mathrm{~K}$, the peak of the absorption spans from few tens up to several hundreds $\mathrm{GeV}$. The combination of all these effects will result in a strong modulation of the $\gamma-\gamma$ absorption around periastron, as shown in Fig. 4. A modification of the black-body emission by absorption in the wind will move the peak of the $\gamma-\gamma$ absorption toward even higher energies.

A large combination of parameters is possible, but quality of current data (Fermi-LAT and H.E.S.S.) do not allow to refine any model parameter. The better CTA sensitivity (many orders of magnitude) with respect to Fermi-LAT, in particular for very short observations [39], will allow to decrease enormously the temporal binning of the LC, offering an unprecedented level of details and $\mathrm{S} / \mathrm{N}$ for variability studies and spectral analyses.

In Fig. 3 a 50 hours CTA simulation of an arbitrary spectrum around periastron is reported. It 
has been obtained using the CTOOLS and Prod3b-v2 IRF of the South array ${ }^{4}$, publicly available $^{5}$. Provided that the loosely constrained optical depth is in a reasonable range, the $\pi^{0}$ decay continuum could optimistically be detected even with very short exposure of $\lesssim 1$ hour.

A detailed study of the $\eta$ Carinae LC variability and the VHE cut off modulation will allow to probe the geometry of the system, the magnetic field configuration, the location where protons are accelerated and their maximum energy, as well as the distribution of the soft photons in a very complex environment [40]. Finally, the study of $\eta$ Carinae offers a remarkable opportunity to get a close-up of one of the most massive stars known during the final stage of its evolution, as well as the strong shock wind interactions with its companion.

\section{References}

[1] D. C. Abbott and P. S. Conti, Wolf-rayet stars., Annual Review of Astronomy and Astrophysics 25 (1987) 113.

[2] P. S. Conti, Mass loss in early-type stars., Annual Review of Astronomy and Astrophysics 16 (1978) 371.

[3] M. De Becker, Non-thermal emission processes in massive binaries, Astronomy and Astrophysics Review 14 (2007) 171 [0709.4220].

[4] D. Eichler and V. Usov, Particle Acceleration and Nonthermal Radio Emission in Binaries of Early-Type Stars, Astrophysical Journal 402 (1993) 271.

[5] P. Benaglia and G. E. Romero, Gamma-ray emission from Wolf-Rayet binaries, Astronomy and Astrophysics 399 (2003) 1121 [astro-ph/ 0205375 ].

[6] D. C. Abbott, J. H. Beiging, E. Churchwell and A. V. Torres, Radio Emission from Galactic Wolf-Rayet Stars and the Structure of Wolf-Rayet Winds, Astrophysical Journal 303 (1986) 239.

[7] M. De Becker and F. Raucq, Catalogue of particle-accelerating colliding-wind binaries, Astronomy and Astrophysics 558 (2013) A28 [1308.3149].

[8] D. J. Hillier, K. Davidson, K. Ishibashi and T. Gull, On the Nature of the Central Source in $\eta$ Carinae, Astrophysical Journal 553 (2001) 837.

[9] R. M. Humphreys, K. Davidson and N. Smith, $\eta$ Carinae's Second Eruption and the Light Curves of the $\eta$ Carinae Variables, Publications of the Astronomical Society of the Pacific 111 (1999) 1124.

[10] K. Davidson and R. M. Humphreys, Eta Carinae and Its Environment, Annual Review of Astronomy and Astrophysics 35 (1997) 1.

[11] N. Smith and D. J. Frew, A revised historical light curve of Eta Carinae and the timing of close periastron encounters, Monthly Notices of the RAS 415 (2011) 2009 [1010 . 3719].

[12] H. L. Gomez, C. Vlahakis, C. M. Stretch, L. Dunne, S. A. Eales, A. Beelen et al., Submillimetre variability of Eta Carinae: cool dust within the outer ejecta, Monthly Notices of the RAS 401 (2010) L48 [0911.0176].

[13] N. Smith, R. D. Gehrz, P. M. Hinz, W. F. Hoffmann, J. L. Hora, E. E. Mamajek et al., Mass and Kinetic Energy of the Homunculus Nebula around $\eta$ Carinae, Astronomical Journal 125 (2003) 1458.

\footnotetext{
${ }^{4}$ I used the South_z40_50h IRF.

${ }^{5}$ They can be downloaded from: https://www.cta-observatory.org/science/cta-performance
} 
[14] A. Damineli, The 5.52 Year Cycle of Eta Carinae, Astrophysical Journal 460 (1996) L49.

[15] J. H. Groh, D. J. Hillier, T. I. Madura and G. Weigelt, On the influence of the companion star in Eta Carinae: $2 D$ radiative transfer modelling of the ultraviolet and optical spectra, Monthly Notices of the RAS 423 (2012) 1623 [1204.1963].

[16] J. M. Pittard and M. F. Corcoran, In hot pursuit of the hidden companion of eta Carinae: An X-ray determination of the wind parameters, Astronomy and Astrophysics 383 (2002) 636 [astro-ph/0201105].

[17] H. V. Bradt, R. E. Rothschild and J. H. Swank, X-ray timing explorer mission, Astronomy and Astrophysics Supplement Series 97 (1993) 355.

[18] A. Damineli, D. J. Hillier, M. F. Corcoran, O. Stahl, R. S. Levenhagen, N. V. Leister et al., The periodicity of the $\eta$ Carinae events, Monthly Notices of the RAS 384 (2008) 1649 [0711.4250].

[19] A. T. Okazaki, S. P. Owocki, C. M. P. Russell and M. F. Corcoran, Modelling the RXTE light curve of $\eta$ Carinae from a 3D SPH simulation of its binary wind collision, Monthly Notices of the RAS 388 (2008) L39 [0 805 . 1794].

[20] E. R. Parkin, J. M. Pittard, M. F. Corcoran and K. Hamaguchi, Spiraling Out of Control: Three-dimensional Hydrodynamical Modeling of the Colliding Winds in $\eta$ Carinae, Astrophysical Journal 726 (2011) 105 [1011.0778].

[21] I. R. Stevens, J. M. Blondin and A. M. T. Pollock, Colliding Winds from Early-Type Stars in Binary Systems, Astrophysical Journal 386 (1992) 265.

[22] M. F. Corcoran, K. Hamaguchi, J. K. Liburd, D. Morris, T. R. Gull, T. I. Madura et al., The X-ray Lightcurve of the Supermassive star eta Carinae, 1996-2014, arXiv e-prints (2015) arXiv:1507.07961 [1507.07961].

[23] R. Dgani, R. Walder and H. Nussbaumer, Stability analysis of colliding winds in a double star system., Astronomy and Astrophysics 267 (1993) 155.

[24] D. Folini and R. Walder, 3D Hydrodynamical Simulations of Colliding Wind Binaries: Theory Confronts Observations, Astrophysics and Space Science 274 (2000) 189.

[25] J.-C. Leyder, R. Walter and G. Rauw, Hard X-ray emission from $\eta$ Carinae, Astronomy and Astrophysics 477 (2008) L29 [0 712 . 1491].

[26] M. Balbo and R. Walter, Fermi acceleration along the orbit of $\eta$ Carinae, Astronomy and Astrophysics 603 (2017) A111 [1705.02706].

[27] E. Leser, S. Ohm, M. Füßling, M. de Naurois, K. Egberts, P. Bordas et al., First Results of Eta Carinae Observations with H.E.S.S. II, International Cosmic Ray Conference 301 (2017) 717 [1708.01033].

[28] A. Reimer, M. Pohl and O. Reimer, Nonthermal High-Energy Emission from Colliding Winds of Massive Stars, Astrophysical Journal 644 (2006) 1118 [astro-ph/ 0510701 ].

[29] C. Panagiotou and R. Walter, The environment of the wind-wind collision region of $\eta$ Carinae, Astronomy and Astrophysics 610 (2018) A37 [1712.01382].

[30] D. Falceta-Gonçalves and Z. Abraham, MHD numerical simulations of colliding winds in massive binary systems - I. Thermal versus non-thermal radio emission, Monthly Notices of the RAS 423 (2012) 1562 [1203.5093]. 
[31] G. Kowal, E. M. de Gouveia Dal Pino and A. Lazarian, Magnetohydrodynamic Simulations of Reconnection and Particle Acceleration: Three-dimensional Effects, Astrophysical Journal 735 (2011) 102 [1103.2984].

[32] G. Kowal, A. Lazarian, E. T. Vishniac and K. Otmianowska-Mazur, Numerical Tests of Fast Reconnection in Weakly Stochastic Magnetic Fields, Astrophysical Journal 700 (2009) 63 [0903.2052].

[33] J. Park, D. Caprioli and A. Spitkovsky, Simultaneous Acceleration of Protons and Electrons at Nonrelativistic Quasiparallel Collisionless Shocks, Physical Review Letters 114 (2015) 085003 [1412.0672].

[34] S. Ohm, V. Zabalza, J. A. Hinton and E. R. Parkin, On the origin of $\gamma$-ray emission in $\eta$ Carina, Monthly Notices of the RAS 449 (2015) L132 [1502 . 04056 ].

[35] K. Reitberger, O. Reimer, A. Reimer, M. Werner, K. Egberts and H. Takahashi, Gamma-ray follow-up studies on $\eta$ Carinae, Astronomy and Astrophysics 544 (2012) A98 [1203. 4939].

[36] G. Voisin, F. Mottez and S. Bonazzola, Electron-positron pair production by gamma-rays in an anisotropic flux of soft photons, and application to pulsar polar caps, Monthly Notices of the RAS 474 (2018) 1436 [1710.04021].

[37] T. I. Madura, T. R. Gull, S. P. Owocki, J. H. Groh, A. T. Okazaki and C. M. P. Russell, Constraining the absolute orientation of $\eta$ Carinae's binary orbit: a 3D dynamical model for the broad [Fe III] emission, Monthly Notices of the RAS 420 (2012) 2064 [1111.2226].

[38] M. Balbo and R. Walter, $\gamma-\gamma$ opacity study in $\eta$ Carinae, in preparation (2019) .

[39] S. Funk, J. A. Hinton and CTA Consortium, Comparison of Fermi-LAT and CTA in the region between 10-100 GeV, Astroparticle Physics 43 (2013) 348 [1205. 0832].

[40] M. Chernyakova, D. Malyshev, A. Paizis, N. La Palombara, M. Balbo, R. Walter et al., Overview of non-transient gamma-ray binaries and prospects for the Cherenkov Telescope Array, accepted to Astronomy and Astrophysics (2019) [1909.11018]. 